\title{
Method of the Determination of Exterior Orientation of Sensors in Hilbert Type Space ${ }^{+}$
}

\author{
Grzegorz Stępień \\ Institute of Geoinformatics, Żołnierska 46, Maritime University of Szczecin, 71-250 Szczecin, Poland; \\ g.stepien@am.szczecin.pl \\ + Presented at the 5th International Symposium on Sensor Science (I3S 2017), Barcelona, Spain, \\ 27-29 September 2017.
}

Published: 1 December 2017

The following article presents the new algorithm of the isometric transformation based on the transformation in the newly normed Hilbert type space. The presented method is based on so-called virtual translations, already known in advance, of two relative oblique orthogonal coordinate systems - interior and exterior orientation of sensors - to a common, known in both systems points. Each of the systems is translated along its axis (the systems have common origins) and at the same time the angular relative orientation of both coordinate systems is constancy. The translation of both coordinate systems is defined by the spatial norm determining the length of vectors in the new Hilbert type space. As such, the displacement of two relative oblique orthogonal systems is reduced to zero. This makes it possible to directly calculate the rotation matrix of the sensor. The next and final step is the return translation of the system along an already known track. The method can be used for big rotation angles. The method was verified in laboratory conditions for the test data set and measurement data (field data). The accuracy of the results in the laboratory test is on the level of $10^{-6}$ of the input data. This confirmed the correctness of the assumed calculation method. The method is the further development of the Total Free Station (TFS) transformation (Stępien et al., 2017) to several centroids in Hilbert type space. This is the reason why the method is called Multi-Centroid Isometric Transformation-MCIT. MCIT is very fast and enables, by reducing to zero the translation of two relative oblique orthogonal coordinate systems, direct calculation of the exterior orientation of the sensors.

(C) 2017 by the authors. Licensee MDPI, Basel, Switzerland. This article is an open access article distributed under the terms and conditions of the Creative Commons Attribution (CC BY) license (http://creativecommons.org/licenses/by/4.0/). 